\title{
On Arithmetic Functions in Actin Filament Networks
}

\author{
Andrew Schumann \\ University of Information Technology and \\ Management in Rzeszow \\ 35-225 Rzeszow \\ Poland \\ andrew.schumann@gmail.com
}

\begin{abstract}
This paper is devoted to actin filament networks as a computation medium. The point is that actin filaments are sensitive to outer cellular stimuli (attractants as well as repellents) and they appear and disappear at different places of the cell to change the cell structure, e.g. its shape. Due to assembling and disassembling actin filaments, Amoeba proteus can move in responses to different stimuli. As a result, Amoeba proteus can be considered a simple reversible logic gate, where outer cellular signals are its inputs and the amoeba motions are its outputs. In this way, we can implement the FREDKIN logic gate on the amoeba behaviours. The actin filament networks have the same basic properties as neural networks: lateral inhibition; lateral activation; recurrent inhibition; recurrent excitation; feedforward inhibition; feedforward excitation; convergence/divergence. These networks can embody arithmetic functions defined recursively and corecursively within p-adic valued logic. Furthermore, within these networks we can define the so-called diagonalization for deducing undecidable arithmetic functions.
\end{abstract}

\section{CCS Concepts}

-Computing methodologies $\rightarrow$ Parallel computing methodologies; Artificial intelligence; $\bullet$ Parallel algorithms $\rightarrow$ Massively parallel algorithms; •Artificial intelligence $\rightarrow$ Philosophical/theoretical foundations of artificial intelligence;

\section{Keywords}

Actin filament; F-actin; G-actin; p-adic integer; neural network; p-adic valued logic; corecursion; diagonalization

\section{INTRODUCTION}

Physarum polycephalum is one of the unicellular organisms best studied from the standpoint of computation theory [2], [19], [21], [22]. In the project Physarum Chip Project: personal or classroom use is granted without fee provided that copies

Permission to make digital or hard copies of all or part of this work for personal or classroom use is granted without fee provided that copies are not made or distributed for profit or commercial advantage and that copies bear this notice and the full citation on the first page. To copy otherwise, to republish, to post on servers or to redistribute to lists, requires prior specific permission and/or a fee. BICT 2017, March 15-16, Hoboken, United States

ISBN 978-1-63190-148-5

DOI: 10.4108/eai.22-3-2017.152402

Copyright $\odot 2017$ EA
Growing Computers From Slime Mould supported by FP7 and organized by Andrew Adamatzky, we have designed some processors on the basis of the Physarum polycephalum motions. The plasmodium of Physarum polycephalum consists mainly from actin filament networks and these networks are responsible for the intelligent behaviour of Physarum polycephalum [11].

In this paper I show that any cell can be considered a computer due to its actin filament networks, in particular Amoeba proteus can be regarded as a logic gate (Sections 2,3 ). The intelligent properties of neural networks such as lateral inhibition or recurrent excitation are also connected to appropriate properties of actin filament networks (Section $4)$. In actin filament networks we can define decidable and undecidable arithmetic functions (Sections 5, 6).

\section{MOTILITY OF AMOEBA PROTEUS AND ACTIN FILAMENTS}

Amoeba proteus (see Fig.1) is very sensitive to the environment and reacts directly to external stimuli by the motility of its shape. This shape changes due to the cytoplasmic streaming that extends pseudopodia towards attractants (food). So, the amoeboid locomotion is committed forward if the amoeba detects an attractant. Meanwhile, for A. proteus there exist repellents, as well: the amoeba avoids strong light (and it moves towards the weaker light), also it avoids dark (it moves towards light) and many other conditions: some chemicals (such as salt), obstacles, anode (it moves towards cathode), cold (it moves towards soft), and hot (it prefers soft), etc.

The amoeboid reactions to attractants and repellents are studied well and explained by actin filaments or F-actin (see Fig.2), i.e. the protein which is organized into higherorder structures, forming linear bundles, two-dimensional networks, and three-dimensional semisolid gels. Actin monomers polymerize to form thin, flexible fibers (actin filaments) 5-9 $\mathrm{nm}$ in diameter and up to several micrometers in length. Actin filaments are connected to the plasma membrane, where they form an actin cortex that provides mechanical support (see Fig.3). If there is an attractant before the cell, actin filaments form a wave to change the cell shape to allow the movement of the cell surface to build a pseudopodia by cross-linked filaments (see Fig.3).

Actin filaments under different external conditions can be assembled and disassembled and these reactions are regulated by actin-binding proteins. For instance, on the one hand, cofilin remains bound to actin monomers following filament disassembly and sequesters them in the ADP-bound 


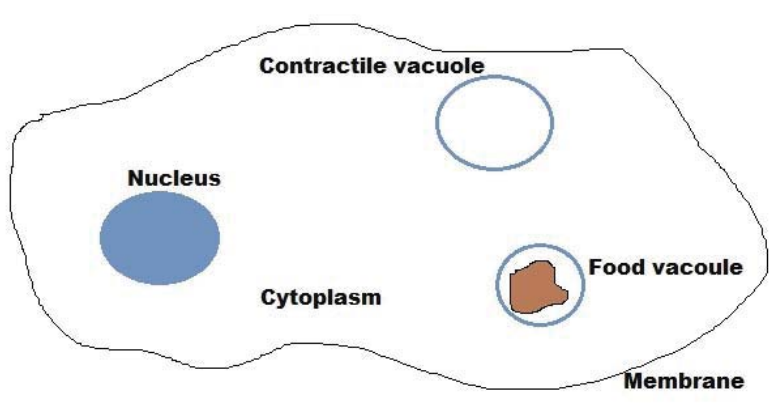

Figure 1: The morphology of Amoeba proteus: (1) contractile vacuole (a water bubble within the endoplasm of $A$. proteus to regulate the water content of the cell); (2) nucleus (a membrane bound organelle containing the cell's genetic information and responsible for the actions of the amoeba); (3) cytoplasm (a gel-like substance that allows $A$. proteus to form its pseudopodia and preform its respective functions, it contains actin filaments which are responsible for sensitivity of $A$. proteus); (4) food vacuole (a vacuole with a digestive function, containing a food for $A$. proteus); (5) membrane (it contains the inner part of the cell such as organelles and cytoplasm, it has good regenerative abilities and elasticity).

form. On the other hand, profilin stimulate the incorporation of actin monomers into filaments. Also, there are actinbinding proteins connecting two different actin filaments into bundles or even into networks which can crosslink perpendicular filaments.

First of all, actin filaments form the cell cortex, which lies adjacent to the plasma membrane to support it (Fig.4). This cytoskeleton is dynamic and sensitive to the cell surroundings. Each external force (each taxis) acting on the actin cortex are transmitted by signaling pathways to directly react to the external environment. If actin filaments are assembled in parallel with the same polarity direction, they propagate some projections, called microvilli, by adding new monomers at the plus ends adjacent to the plasma membrane (Fig.5). For the cell migration actin filaments are crosslinked to propagate membrane protrusions in the form of filopodia (Fig.6) or lamellipodia (Fig.7). They are being formed also to probe the cell microenvironment. The more stable bundles of actin filaments are represented by stress fibers (Fig.8) which allow the cell to form a track system for cargo transport. In the cell they build up networks which change their topology by reactions to the external forces.

Hence, actin filaments are instable, they can assemble and disassemble rapidly by polymerization and depolymerization respectively. For more details see [4], [5], [6], [7], [9], [10], [12], [13], [14], [15], [16].

\section{MOTILITY OF AMOEBA PROTEUS AND THE FREDKIN GATE}

Taking into account the fact that it is known in general how it is possible to control the polymerization and depolymerization of actin filaments, we can consider the amoeba

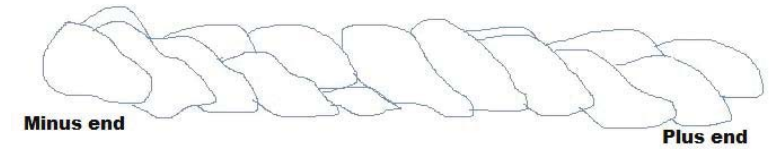

Figure 2: The actin filament or F-actin is a linear polymer of globular actin monomers (G-actin). Factin is flexible and has a helical repeat every $37 \mathrm{~nm}$. It ranges from 5-9 $\mathrm{nm}$ in diameter. It has a rotation of $166.15^{\circ}$ around the axis. Each G-actin has tight binding sites that mediate head-to-tail interactions with two other actin monomers, in this way actin monomers are oriented in the same direction and their polymerization gives a distinct polarity at the ends of the actin filament: the plus and minus ends. At these ends there are different rates of the actin filament grow so that we have the plus end to which monomers are added five to ten times faster than to the slow-growing minus end.

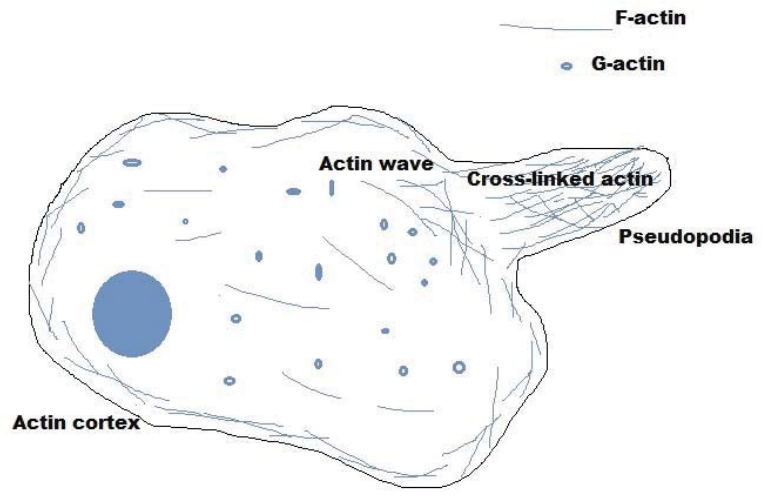

Figure 3: Actin filaments responsible for the motility of Amoeba proteus.

(more correctly, the actin filament networks) as an automaton, where (i) the external forces, to which the actin filaments are responding, are its inputs and (ii) all the responses causing the amoeba motility are its outputs. In this way it is easier to implement reversible logic gates, where a unique input is associated with a unique output and vice versa. In these gates, the automaton maps each distinct bit string input of the length $n$ into a distinct bit string output of the same length. For example, the FREDKIN gate is reversible, for the input $[A G E]$ it gives the output $[C D B]$ by the following rule: $A=C, D=\mathrm{OR}(\operatorname{AND}(\operatorname{NOT} A, G), \operatorname{AND}(A, E))$, and $B=\operatorname{OR}(\operatorname{AND}(A, G), \operatorname{AND}(\operatorname{NOT} A, E))$, i.e. we deal with the three inputs $A, G, E$, and the three outputs $C$, $D, B$. See Table 1 .

The FREDKIN gate can be implemented by the Amoeba proteus motility as follows. Let us take the input string $[A G E]$ with the following meaning: (i) $A=0$ if an appropriate zone of the microenvironment contains an obstacle (barrier) and $A=1$ otherwise; (ii) $G=1$ if an appropriate zone of the microenvironment contains an attractant and $G=0$ otherwise; (iii) $E=0$ if an appropriate zone of the microenvironment contains an obstacle (barrier) and $E=1$ 


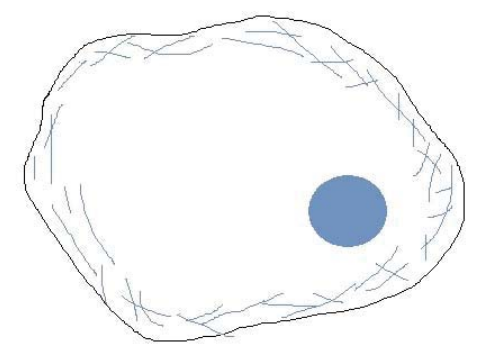

Figure 4: The cell cortex of Amoeba proteus.

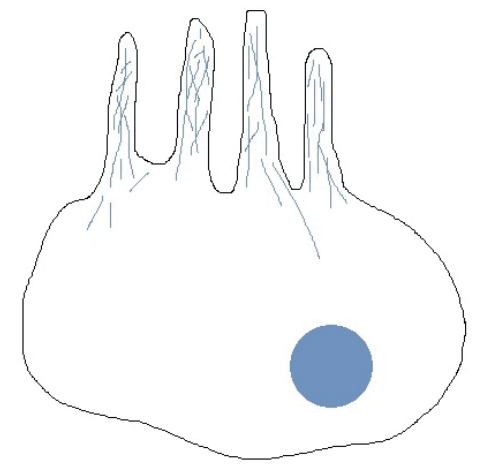

Figure 5: The microvilli of Amoeba proteus.

otherwise. Let us assume that the output string $[C D B]$ with the following meaning: (i) $C=1$ if an appropriate zone of the cell has a deformation because of assembling an actin filament network and $C=0$ otherwise; (ii) $D=1$ if an appropriate zone of the cell has a deformation because of assembling an actin filament network and $D=0$ otherwise; (iii) $B=1$ if an appropriate zone of the cell has a deformation because of assembling an actin filament network and $B=0$ otherwise.

Thus, we can implement some reversible logic gates on the amoeboid motions based on actin filament networks.

We can define now an Euclidean cellular automaton [8] over a parameter space $P=[0,1]^{n}$ presented by the inputs $\left(i_{1}, i_{2}, \ldots, i_{n}\right)$ (i.e. external forces acting on actin filaments) and the outputs $\left(o_{1}, o_{2}, \ldots, o_{n}\right)$ (i.e. reactions of actin filament networks). This automaton is defined as a 4-tuple

\begin{tabular}{ccccccccc}
\hline & $\mathbf{0 0 0}$ & $\mathbf{0 0 1}$ & $\mathbf{0 1 0}$ & $\mathbf{0 1 1}$ & $\mathbf{1 0 0}$ & $\mathbf{1 0 1}$ & $\mathbf{1 1 0}$ & $\mathbf{1 1 1}$ \\
\hline $\mathbf{0 0 0}$ & 1 & 0 & 0 & 0 & 0 & 0 & 0 & 0 \\
$\mathbf{0 0 1}$ & 0 & 1 & 0 & 0 & 0 & 0 & 0 & 0 \\
$\mathbf{0 1 0}$ & 0 & 0 & 1 & 0 & 0 & 0 & 0 & 0 \\
$\mathbf{0 1 1}$ & 0 & 0 & 0 & 1 & 0 & 0 & 0 & 0 \\
$\mathbf{1 0 0}$ & 0 & 0 & 0 & 0 & 1 & 0 & 0 & 0 \\
$\mathbf{1 0 1}$ & 0 & 0 & 0 & 0 & 0 & 0 & 1 & 0 \\
$\mathbf{1 1 0}$ & 0 & 0 & 0 & 0 & 0 & 1 & 0 & 0 \\
$\mathbf{1 1 1}$ & 0 & 0 & 0 & 0 & 0 & 0 & 0 & 1 \\
\hline
\end{tabular}

Table 1: The FREDKIN gate in the permutation matrix form. The input [000] is mapped to the output $[000],[001]$ is mapped to [001], etc.

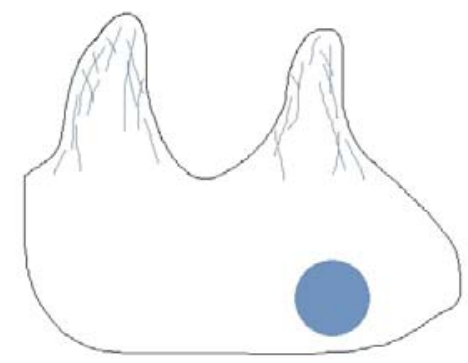

Figure 6: The filopodia of Amoeba proteus.

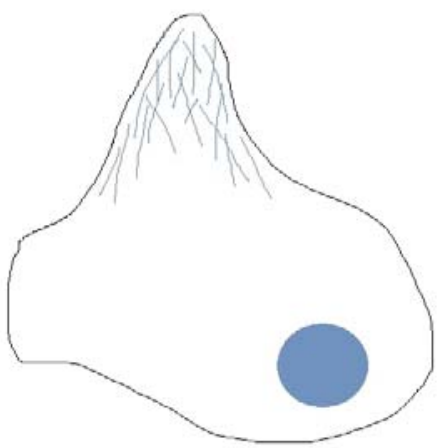

Figure 7: The lamellipodium of Amoeba proteus.

$(\mathcal{P}, I, F, T)$ where $\mathcal{P} \subset 2^{P}$ is a finite set of states of actin filament networks given as subsets of $P ; I \subset \mathcal{P}$ is the set of initial states; $F \subset \mathcal{P}$ is the set of accepting states; and $T: P \times \mathcal{P} \rightarrow \mathcal{P}$ is the transition function that assigns for each parameter setting $\mathbf{v}=\left(i_{1}, i_{2}, \ldots, i_{n}\right) \in P$ and each state $\mathbf{s} \in \mathcal{P}$ a next state $\mathbf{t}=T(\mathbf{v}, \mathbf{s})$. The parameter $\mathbf{v} \in P$ is defined as a neighborhood for $\left(o_{1}, o_{2}, \ldots, o_{n}\right)$ with a radius $\epsilon: \mathbf{v}=\left\{\left(i_{1}, i_{2}, \ldots, i_{n}\right):\left|i_{k}-o_{k}\right| \leq \epsilon, k=\overline{1, n}\right\}$. In this automaton we deal with a continuous domain and with a finite set of states, i.e. with subsets $P_{i}$ of $P$ indexed from a finite index set $S$. If $P_{i} \cap P_{j}=\emptyset$ for all $i, j \in S$ we call the Euclidian cellular automaton deterministic, if $\bigcup_{i \in S} P_{i}=P$ we call it complete.

In the FREDKIN gate, the parameter space $P=[0,1]^{3}$. The input $\left(i_{1}, i_{2}, i_{3}\right)$ shows the localizations of attractants and repellents in the FREDKIN motions of the amoeba and the output $\left(o_{1}, o_{2}, o_{3}\right)$ shows the localization of the amoeba cell in appropriate FREDKIN motions. Using this gate in the way of [18], we can implement the adder on Amoeba proteus and then some arithmetic functions.

\section{ACTIN FILAMENT NETWORKS}

An actin filament network is defined as a system $\left(N^{\prime}, V^{\prime}\right.$, $w^{\prime}$ ), where (i) $N^{\prime}$ is a non-well-founded set of processors called 'filaments'; this set is non-well-founded, because it is impossible to divide $N^{\prime}$ into atoms or even just into excluded subsets $n_{j}$ which form a partition of $N^{\prime}=\bigsqcup_{j} n_{j}$; in other words, processors are being redesigned permanently and they can appear and disappear and ever change own features; (ii) $V^{\prime}$ is a set of tuples $\left\{\left(i_{t}, j_{t}\right): i_{t}, j_{t} \in N^{\prime}\right\}$ whose elements are connections between filament $i_{t}$ and filament 


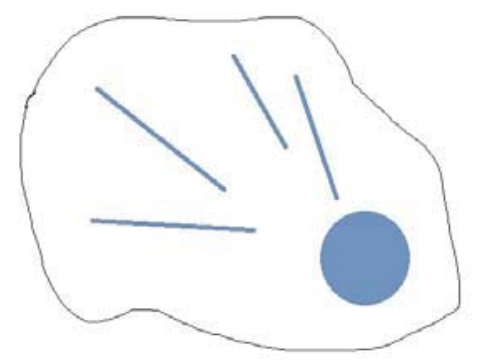

Figure 8: The stress fibers of Amoeba proteus.

\section{$A=1$}

$E=1$

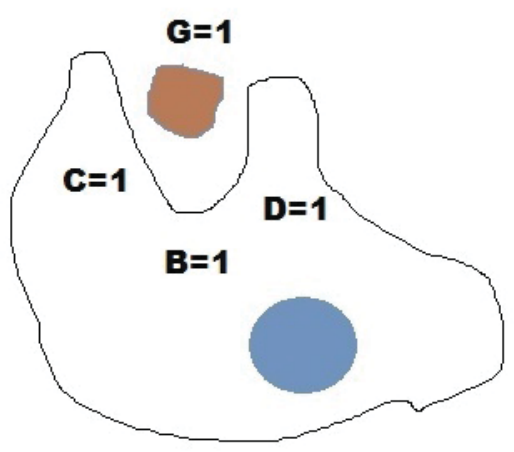

Figure 9: The FREDKIN gate for Amoeba proteus with the three inputs $A=1, G=1, E=1$ and the three outputs $C=1$ (the cell zone $C$ moves forward), $D=1$ (the cell zone $D$ moves forward), $B=1$ (the cell zone $B$ moves backward).

$j_{t}$ at time step $t$; hence, the set $V^{\prime}$ is non-well-founded, too, as its cardinality can change during the time $t$; (iii) $w^{\prime}$ is a function from $V^{\prime}$ to ${ }^{*} \mathbf{R}$, where ${ }^{*} \mathbf{R}$ is a set of hyperreal numbers such that $w^{\prime}\left(\left({ }^{*} i,{ }^{*} j\right)\right)$, where ${ }^{*} i=i_{0} i_{1} i_{2} i_{3}, \ldots$ and ${ }^{*} j=j_{0} j_{1} j_{2} j_{3} \ldots$, for short $w{ }^{*}{ }_{i,}{ }^{j} j$, is called the weight of the connection between filament ${ }^{*} i$ and filament ${ }^{*} j$ at each time step $t=0,1,2,3, \ldots$; notice that a filament ${ }^{*} i$ can be hidden (not present) at actual time.

This network is more general than artificial neural networks. The main difference is that in the latter the processors ('neurons') do not disappear, because they are fixed, but in the actin filament networks the processors ('filaments') appear and disappear permanently.

It is worth noting that the actin filaments are responsible for remodeling neurons in many-cellular organisms possessing the nervous system, also. In this system the actin filaments change the shape and structure of dendritic spines in the same way as they do it for the amoeba motility. G-actin is distributed throughout the whole axon and the whole dendrite and it can be polymerized into F-actin to form new spines as well as to stabilize the spine volume. As a result, the actin filaments form new synapses to increase the cell communication. The filament polymerization promotes

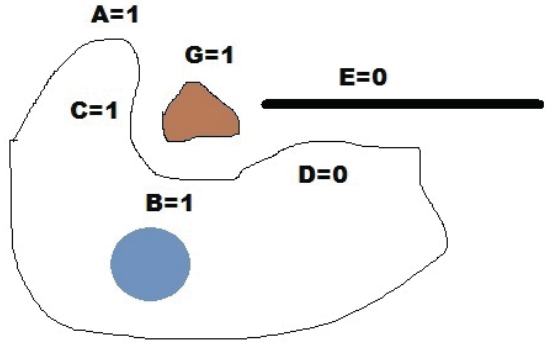

Figure 10: The FREDKIN gate for Amoeba proteus with the three inputs $A=1, G=1, E=0$ and the three outputs $C=1$ (the cell zone $C$ moves forward), $D=0$ (the cell zone $D$ does not move), $B=1$ (the cell zone $B$ moves backward).

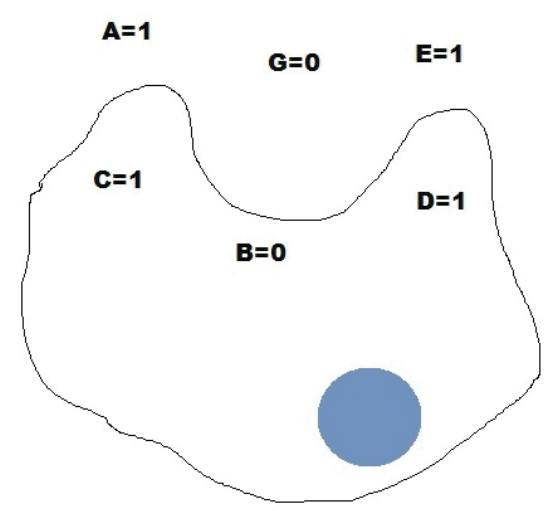

Figure 11: The FREDKIN gate for Amoeba proteus with the three inputs $A=1, G=0, E=1$ and the three outputs $C=1$ (the cell zone $C$ moves forward), $D=1$ (the cell zone $D$ moves forward), $B=0$ (the cell zone $B$ does not move).

long-term potentiation increasing the spine volume and the cell communication. The filament depolymerization leads to a long-term depression decreasing the spine volume and the cell communication.

In the actin filament networks we find out all the basic properties of the neural networks [17]:

- Lateral inhibition. In neurons, a presynaptic cell excites inhibitory interneurons and they inhibit neighboring cells in the neural network. As a result, the contrast of the signal is made more visible. In actin filaments, neighboring bundles are inhibited to increase the intensity of the signal.

- Lateral activation. In neurons, a presynaptic cell excites activation interneurons and they activate neighboring cells in the neural network. As a consequence, the contrast of the signal is made less visible. In actin filaments, neighboring bundles are activated to decrease the intensity of the signal.

- Feedback/recurrent inhibition. In neurons, a presynaptic cell transmits the signal to a postsynaptic cell, and 


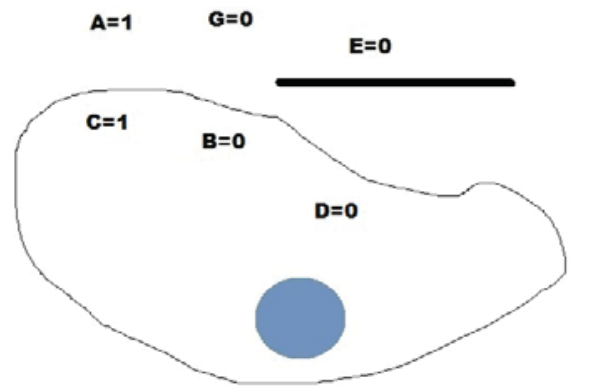

Figure 12: The FREDKIN gate for Amoeba proteus with the three inputs $A=1, G=0, E=0$ and the three outputs $C=1$ (the cell zone $C$ moves forward), $D=0$ (the cell zone $D$ does not move), $B=0$ (the cell zone $B$ does not move).

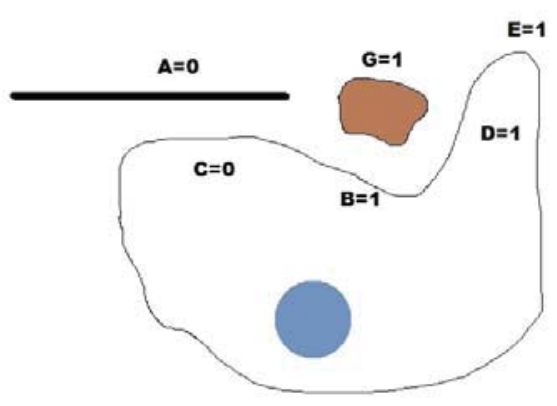

Figure 13: The FREDKIN gate for Amoeba proteus with the three inputs $A=0, G=1, E=1$ and the three outputs $C=0$ (the cell zone $C$ does not move), $D=1$ (the cell zone $D$ moves forward), $B=1$ (the cell zone $B$ moves backward).

the postsynaptic cell in turn transmits it to an interneuron, which then inhibits the presynaptic cell. Due to this circuit there is a limitation for the excitation and the rhythmic changing in the transmission of the signal is possible. The same takes place for the actin filament bundles causing the generating of rhythmic behaviors.

- Feedback/recurrent excitation. A presynaptic cell excites a postsynaptic neuron and the postsynaptic neuron excites in turn the presynaptic cell. It is used for learning and memory processes. In actin filaments, recurrent excitation accumulates the external stimuli as a positive feedback to continue the same pattern of behaviour.

- Feedforward inhibition. A presynaptic neuron excites an inhibitory interneuron that inhibits the next neuron. The actin filaments ignore some signals.

- Feedforward excitation. A presynaptic neuron excites a postsynaptic neuron. In actin filaments, we have a direct action in changing the actin filaments caused by one external stimulus.

- Convergence/Divergence. A postsynaptic neuron re-

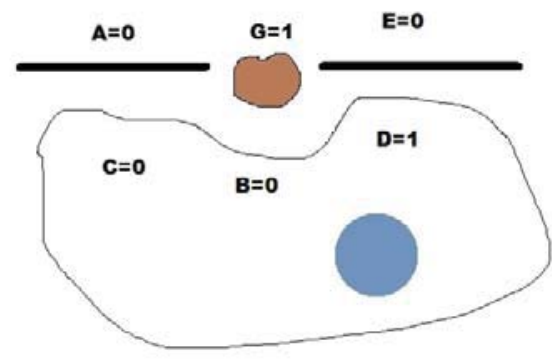

Figure 14: The FREDKIN gate for Amoeba proteus with the three inputs $A=0, G=1, E=0$ and the three outputs $C=0$ (the cell zone $C$ does not move), $D=1$ (the cell zone $D$ moves right), $B=0$ (the cell zone $B$ does not move).

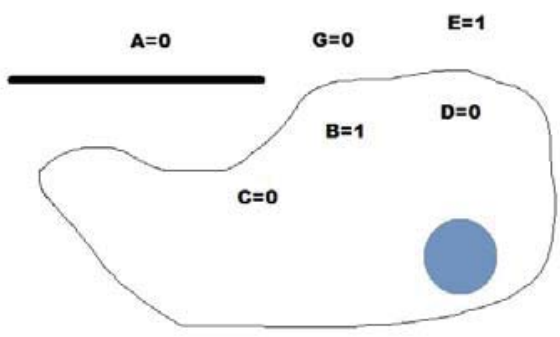

Figure 15: The FREDKIN gate for Amoeba proteus with the three inputs $A=0, G=0, E=1$ and the three outputs $C=0$ (the cell zone $C$ does not move), $D=0$ (the cell zone $D$ does not move), $B=1$ (the cell zone $B$ moves forward).

ceives a convergent input from a number of different presynaptic neurons and this postsynaptic neuron makes further divergent connections to other postsynaptic neurons. Convergence allows a cell to receive a signal from many cells and divergence allows a cell to transmit the signal further. Secreting a cyst wall of the amoeba is an example of this effect for the actin filament bundles in Amoeba proteus.

As we see, the actin filament networks are more complex than neural networks and the basic neuronal properties are connected to appropriate properties of actin filaments. Nevertheless, the actin filament networks are not studied well recently from the point of view of mathematics.

\section{P-ADIC VALUED ARITHMETIC FUNC- TIONS IN ACTIN FILAMENT NETWORKS}

Let us consider the discrete time $t=0,1,2, \ldots$ assuming that at each time step $t$ the actin filaments of the amoeba face not more than $n$ attractants or repellents and react to $n$ stimuli. Then the amoeba motion can be examined as an arithmetic function $f_{2^{n}}(x)=y$, where $x, y \in\left\{0,1, \ldots, 2^{n}-\right.$ $1\}$, e.g. in the Fredkin gate (Fig. $9-16$ ) we deal with the arithmetic function $f_{23}$, where the inputs and the outputs of Table 1 are rewritten as natural numbers: $x_{0} x_{1} x_{2}=$ 


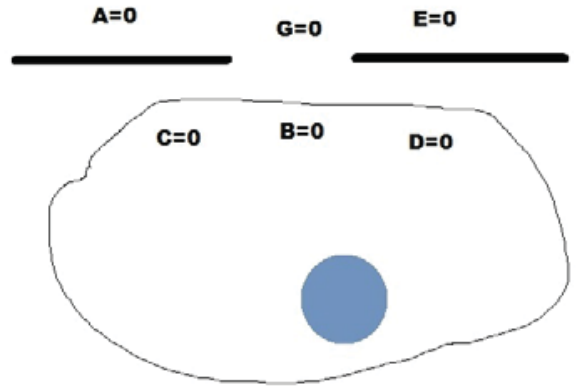

Figure 16: The FREDKIN gate for Amoeba proteus with the three inputs $A=0, G=0, E=0$ and the three outputs $C=0$ (the cell zone $C$ does not move), $D=0$ (the cell zone $D$ does not move), $B=0$ (the cell zone $B$ does not move).

$\sum_{i=0}^{2} x_{i} \cdot 2^{i}$. For example, $000=0$ and $111=\sum_{i=0}^{2} 1 \cdot 2^{i}=7$. Hence, if we have $n$ signals at the time step $t$, then Amoeba proteus calculates an arithmetic function $f_{2^{n}}$ at this $t$. What $f_{2^{n}}$ is in fact, depends on the topology of $n$ stimuli (their intensity, localization, combination, etc.). For instance, the combination of inputs $A G E$, where $G$ is an attractant or its absence and $A E$ are barriers or their absence, gives us the FREDKIN gate.

Thus, if the amoeba meets not more than $n$ stimuli at $t=0,1,2, \ldots$, then we obtain a sequence of functions:

$$
f_{2^{n}}^{t=0} f_{2^{n}}^{t=1} f_{2^{n}}^{t=2} \ldots,
$$

where at each $t=i$ the arithmetic function $f_{2^{n}}^{t=i}$ can be different. Let us denote this sequence by $f$. It can be considered a $p$-adic valued function for $p=2^{n}$ :

$$
f(\alpha)=\beta,
$$

where $\alpha=\alpha_{0} \alpha_{1} \alpha_{2} \ldots$ and $\beta=\beta_{0} \beta_{1} \beta_{2} \ldots$ such that we have $f_{2^{n}}^{t=i}\left(\alpha_{i}\right)=\beta_{i}$ for each $i=0,1,2, \ldots$ The numbers $\alpha$ and $\beta$ are $p$-adic, because

$$
\alpha=\sum_{i=0}^{\infty} \alpha_{i} \cdot p^{i}, \quad \beta=\sum_{i=0}^{\infty} \beta_{i} \cdot p^{i}
$$

and $\alpha_{i}, \beta_{i} \in\{0, \ldots, p-1\}$ for each $i=0,1,2, \ldots$ Hence, the amoeboid long-time locomotion can be simulated by $p$-adic valued arithmetic functions of the form of (2).

In the paper [19] there was proposed the $p$-adic valued logic for simulating the locomotion of Physarum polycephalum plasmodia. The same logic can be used for simulating the Amoeba proteus locomotion as well as actin filament reactions of other cells. In this logic we can combine many trajectories of the form of (1) by which different amoebas have navigated.

Let us notice that arithmetic operations in $p$-adic valued logic can be defined corecursively. Assume that [] is an empty list and a:s is an infinite list of integers from $\{0, \ldots, p-1\}$ with a head a and a tail $\mathbf{s}$. If the tail is a constant, it means that this constant repeats for ever. For example, a:0 means that after a there is an infinite list of 0 . The list $\mathrm{a}: \mathrm{s}$ can be defined as $\mathrm{a}: \mathrm{b}: \mathrm{s}^{\prime}$, the list $\mathrm{a}: \mathrm{b}: \mathrm{s}^{\prime}$ as $a: b: c: s^{\prime}$ ', etc. Meanwhile, $b$ is the first element of the tail s, c is the first element of the tail s', etc. So, each tail

\begin{tabular}{lccccccl}
\hline input strings & \multicolumn{7}{c}{ the number of output string at $t$} \\
\hline$i_{0}$ & $\mathbf{p}-1$ & $p-1$ & $p-1$ & $p-1$ & $p-1$ & $p-1$ & $\ldots$ \\
$i_{1}$ & $p-2$ & $\mathbf{p}-1$ & $p-2$ & $p-1$ & $p-2$ & $p-1$ & $\ldots$ \\
$\ldots$ & $\ldots$ & $\ldots$ & $\ldots$ & $\ldots$ & $\ldots$ & $\ldots$ & $\ldots$ \\
$i_{n}$ & 0 & $p-1$ & 0 & $p-1$ & 0 & $p-1$ & $\ldots$ \\
$i_{n+1}$ & $p-1$ & $p-2$ & $p-1$ & $p-2$ & $p-1$ & $p-2$ & $\ldots$ \\
$\ldots$ & $\ldots$ & $\ldots$ & $\ldots$ & $\ldots$ & $\ldots$ & $\ldots$ & $\ldots$ \\
\hline
\end{tabular}

Table 2: The Cantor's diagonalization showing that the set of all outputs is uncountable in the $p$-adic universe. Let us take the diagonal $(p-1, p-1, p-3, \ldots)$ from the table and change it as follows: $(0,0,1, \ldots)$. This new $p$-adic integer does not occur in the enumeration.

is an infinite list in turn: $\mathbf{s}=\mathrm{b}: \mathbf{s}^{\prime}, \mathbf{s}^{\prime}=\mathrm{c}: \mathbf{s}^{\prime}$ ', etc. Let $\operatorname{next}(\mathrm{a}: \mathrm{b}: \mathrm{s})=\mathrm{b}: \mathrm{s}$. Let us define the $p$-adic valued sum as follows:

$\operatorname{sum}(a, c):[]=\operatorname{sum}(a:[], c:[])$

next $\left(\operatorname{sum}\left(a: b: s, c: d: s^{\prime}\right)\right)=\operatorname{sum}\left(b: s, d: s^{\prime}\right)$

The $p$-adic valued product:

$\operatorname{prod}(\mathrm{a}, \mathrm{c}):[]=\operatorname{prod}(\mathrm{a}:[], \mathrm{c}:[])$

next $\left(\operatorname{prod}\left(a: b: s, c: d: s^{\prime}\right)\right)=\operatorname{sum}\left(\operatorname{prod}\left(a: 0, d: s^{\prime}\right)\right.$, $\left.\operatorname{prod}\left(b: s, c: d: s^{\prime}\right)\right)$

Now, let us assume that $0: 0$ is a minimal $p$-adic integer (it is an infinite list of 0 ) and $p-1: p-1$ is a maximal $p$-adic integer (it is an infinite list of $p-1$ ). Then let us define the $p$-adic valued conjunction corecursively:

$\min (a, c):[]=\min (a:[], c:[])$

next $\left(\min \left(a: b: s, c: d: s^{\prime}\right)\right)=\min \left(b: s, d: s^{\prime}\right)$

as well as $p$-adic valued disjunction:

$\max (a, c):[]=\max (a:[], c:[])$

next $\left(\max \left(a: b: s, c: d: s^{\prime}\right)\right)=\max \left(b: s, d: s^{\prime}\right)$

The negation is defined as follows:

$\operatorname{not}(\mathrm{a}):[]=\mathrm{p}-1-(\mathrm{a}:[])$

next $(\operatorname{not}(a: b: s))=p-1: p-1-(b: s)$

In this way we obtain the ring of $p$-adic integers, $\mathbf{Z}_{p}$, where $p=2^{n}$, with the $p$-adic valued conjunction, disjunction, and negation. For $t \rightarrow \infty$ the amoeba motility implements arithmetic functions on $\mathbf{Z}_{p}$. The field of $p$-adic numbers, $\mathbf{Q}_{p}$, exists just for the prime $p$, but our $p$ is equal to an even number $2^{n}$. So, we cannot obtain the field for $p=2^{n}$, only the ring. Another important feature is that $\mathbf{Z}_{p}$ contains infinite integers and the set $\mathbf{Z}_{p}$ is uncountable (see Table 2). Due to this fact $\mathbf{Z}_{p}$ differs from the ring of integers, $\mathbf{Z}$, a lot (the cardinal number of $\mathbf{Z}_{p}$ is larger, than the cardinal number of $\mathbf{Z}$, i.e. than $\aleph_{0}$ ). In particular, some arithmetic functions on $\mathbf{Z}_{p}$ are undecidable by definition. The matter is that $\mathbf{Z}_{p}$ is a codata set (non-inductive set, i.e. corecursive or coinductive data) with non-Archimedean properties. For $\mathbf{Z}_{p}$, there are no algorithms for calculating all arithmetic functions by definition, because their objects are defined coinductively, not inductively.

Now, we can enumerate all the arithmetic functions which are implementable in actin filament networks as follows. Let us take a partition of all arithmetic functions on $\mathbf{Z}_{p}$ for different $p$ in the way of Table 2. For each $p$ there is the following enumeration of all arithmetic functions for the fixed $t$. Each function $f_{2^{n}}^{t} \in F_{p=2^{n}}^{t}$ is a $t$-th permutation of the numbers 


\begin{tabular}{|c|c|c|c|c|}
\hline & $\begin{array}{c}\text {-st } n(n=1) \\
1 \text {-st } p(p=2)\end{array}$ & $\begin{array}{c}\text { 2-nd } n(n=2) \\
2 \text {-nd } p(p=4)\end{array}$ & $\begin{array}{c}\text { 3-rd } n(n=3) \\
\text { 3-rd } p(p=8)\end{array}$ & $\ldots$ \\
\hline$t=0$ & $f_{2^{1}}^{t=0} \in F_{p=2}^{t=0}$ & $f_{2^{2}}^{t=0} \in F_{p=4}^{t=0}$ & $f_{2^{3}}^{t=0} \in F_{p=8}^{t=0}$ & $\ldots$ \\
\hline$t=1$ & $f_{2^{1}}^{t=1} \in F_{p=2}^{t=1}$ & $f_{2^{2}}^{t=1} \in F_{p=4}^{t=1}$ & $f_{2^{3}}^{t=1} \in F_{p=8}^{t=1}$ & $\cdots$ \\
\hline$t=2$ & $f_{2^{1}}^{t=2} \in F_{p=2}^{t=2}$ & $f_{2^{2}}^{t=} \in F_{p=4}^{t=2}$ & $f_{2^{3}}^{t=2} \in F_{p=8}^{t=2}$ & $\cdots$ \\
\hline$\vdots$ & $\vdots$ & $\vdots$ & $\vdots$ & $\vdots$ \\
\hline$t \rightarrow \infty$ & $f_{2^{1}}^{\infty} \in F_{p=2}^{\infty}$ & $f_{2^{2}}^{\infty} \in F_{p=4}^{\infty}$ & $f_{2^{3}}^{\infty} \in F_{p=8}^{\infty}$ & $\cdots$ \\
\hline
\end{tabular}

Table 3: The partition of the set of all $p$-adic valued arithmetic functions, $F$, into the subsets $F_{p}^{t}$, where $t=0,1,2, \ldots, p=2,4,8,16,32, \ldots$

$\left(0,1, \ldots, 2^{n}-1\right)$ and can be distinguished by the following code

$$
\left\lceil f_{2^{n}}^{t}\right\rceil=\sum_{i=0}^{t}\left(\sum_{j=0}^{2^{n}-2} c_{j i} \cdot j !\right) \cdot\left(\left(2^{n}-1\right) !\right)^{i},
$$

where $c_{j i}$ counts the number of positions in the $i$-th permutation that are to the right of value $j$ and that contain a value less than $j$. For instance, for the FREDKIN gate we have the permutation $(0,1,2,3,4,6,5,7)$. This means that

$\sum_{j=0}^{2^{3}-2} c_{j} \cdot j !=0 \cdot 0 !+0 \cdot 1 !+0 \cdot 2 !+0 \cdot 3 !+0 \cdot 4 !+0 \cdot 5 !+1 \cdot 6 !=$

$$
=720 \text {. }
$$

Let us assume that the FREDKIN gate was applied two times and $t=1$. As a consequence:

$$
\sum_{i=0}^{1}\left(\sum_{j=0}^{2^{3}-2} c_{j i} \cdot j !\right) \cdot\left(\left(2^{3}-1\right) !\right)^{i}=720+720 \cdot 7 !=3629520 .
$$

For the function $f_{2^{n}}^{\infty}$, its code is as follows:

$$
\left\lceil f_{2^{n}}^{\infty}\right\rceil=\sum_{t=0}^{\infty}\left(\sum_{j=0}^{2^{n}-2} c_{j t} \cdot j !\right) \cdot\left(\left(2^{n}-1\right) !\right)^{t} .
$$

This code is $(p-1)$ !-adic.

Hence, each $p$-adic valued arithmetic function (2) coded by (3) denotes just an infinite trajectory of one amoeba under the conditions of not more than $n$ inputs at each time step $t$. These trajectories can be combined by arithmetic and logical operations of the $p$-adic valued logic defined in [19]. In this way we can simulate a colony of amoebas, their common locomotion, their ongoing divisions and deaths.

\section{UNDECIDABLE FUNCTIONS IN ACTIN FILAMENT NETWORKS}

Let us remind that a set $A$ is called computable (decidable or solvable) if there exists a Turing machine $M$ that behaves as follows:

$$
M(x)= \begin{cases}1, & \text { if } x \in A \\ 0, & \text { otherwise }\end{cases}
$$

So, we assume that each $F_{p}^{t}$ is decidable. Let $M_{1}, M_{2}, \ldots$, be a standard list of Turing machines that includes all programs for $F_{p}$ for a fixed $p$ and from the index $i$ it is possible to extract a code $i=\left\lceil f_{2^{n}}^{t}\right\rceil$ such that $M_{i}$ decides that $f_{2^{n}}^{t} \in A$. It means, we suppose that there is a Turing machine $M$ that takes an input $(i, x)$ and gives an output $M_{i}(x)$.

The set $K_{0}=\left\{\langle x, y\rangle: M_{x}(y)\right.$ halts $\}$ is called a halting set. If the computation halts, then we know that $\langle x, y\rangle \in K_{0}$.

\section{TheOREM 1. The set $K_{0}$ is not decidable.}

Proof. We can appeal to diagonalization to prove this statement. Let us assume that $K_{0}$ is decidable and let $M_{0}$ be a Turing machine that decides $K_{0}$. We can define $M_{0}$ as follows:

$$
M_{0}(x)= \begin{cases}1, & M_{x} \text { halts } \\ 0, & \text { otherwise }\end{cases}
$$

Since $M_{0}$ is a Turing machine, it has a code $e$, therefore $M_{e}=M_{0}$. Now, we can define $A=\left\{i: M_{i}(i) \neq 1\right\}$. This set is undecidable for any $i$. Then the set $K_{0} \supseteq\left\{\langle i, i\rangle: M_{i}(i) \neq\right.$ $1\}$ is undecidable, too.

Let us consider an example of $A$ from theorem 1. Take the following machine:

$$
D(M)= \begin{cases}\text { accept, } & M\left(f_{2^{n}}^{t}\right) \text { does not accept } M\left(\left\lceil f_{2^{n}}^{t}\right\rceil\right) ; \\ \text { reject, } & \text { otherwise. }\end{cases}
$$

Then let us define the diagonalization:

$$
D(D)= \begin{cases}\text { accept, } & D(M) \text { does not accept } D(\lceil M\rceil) ; \\ \text { reject, } & \text { otherwise. }\end{cases}
$$

The actin filament networks are too sensitive to the cellular surroundings. We have assumed that the amoeboid motility programmed by the actin filament networks is a kind of the reversible logic gates, i.e. for $n$ inputs it gives just $n$ outputs. However, the situation of the real amoeboid reactions is much more difficult. In reality, it looks like as follows. All the external signals have a scaling that is too different from the actin filament networks. The point is that the networks have a much better zooming than any outer stimulus. This fact allows them to react continuously to all possible signals at any point of the shape. The amoeba is an analog computer.

The latter feature can be formulated as the situation when the number of outputs is larger, than the number of inputs. The amoeboid reactions with the $n$ inputs and the $m>n$ outputs can be considered a hybrid action [20]. The set of hybrid actions can have an infinite set of labels [20]. So, it is unsolvable by definition. The hybrid actions are undecidable in the meaning of theorem 2. Let us define them:

$$
d\left(f_{2^{n}}^{t}\right)= \begin{cases}\text { accept, } & \text { the amoeba accepts } g \text { such that } \\ & g>f_{2^{n}}^{t} \text { for any } f_{2^{n}}^{t} \\ & \text { at } t \text { with the } n \text { inputs; } \\ \text { reject, } & \text { otherwise. }\end{cases}
$$

Thus, we can implement undecidable arithmetic functions in the actin filament networks, too.

\section{DISCUSSION}

The main problem of designing the actin filament networks consists in controlling the signal transmission through the actin filaments. It is known that the actin filaments are involved in signal processing, as well as in memory and learning mechanisms of neuronal cells. The point is that the actin 
filaments support propagation of voltage pulses and, therefore, it is possible to explain the signal transmission through the actin filaments by an interaction between voltage pulses, where 1 ('true') is assigned to the presence of a voltage pulse in a given location of the actin filament, and 0 ('false') is assigned to the pulse's absence, so that Boolean logical gates and a one-bit half-adder with interacting voltage pulses can be constructed well [23].

Furthermore, each actin filament is a double chain of nodes, which take state 0 (resting) or 1 (excited). These states are updated in parallel in discrete time depending on states of two closest neighbours in the node chain and two closest neighbours in the complementary chain. In this way it is possible to represent the actin filaments as an automaton of finite states with transition rules that support traveling and mobile localizations [3], [24], [25]. Also, we can assume that states of nodes depends not only on the current states of neighbouring node but also on their past states so that we assess the effect of memory of past states on the dynamics of acting automata [1].

As we see, there are possible different approaches to formalizing the signal transmission through the actin filaments.

\section{CONCLUSIONS}

The actin filament networks are responsible for cellular intelligent reactions to all the external stimuli. So, if it is possible to create an artificial protein broth which will be a robot solving the complex of various tasks (learning, orientation in space, decision making about transitions etc.), then this broth will consist of actin filaments controlled by us.

\section{REFERENCES}

[1] Alonso-Sanz R., and Adamatzky A. Actin automata with memory, International Journal of Bifurcation and Chaos, 26(1): 1650019, 2016.

[2] Adamatzky A. Physarum Machines: Computers from Slime Mould, World Scientific Series on Nonlinear Science, Series A, 2010.

[3] Adamatzky A., and Mayne R. Actin automata: Phenomenology and localizations, International Journal of Bifurcation and Chaos, 25(2):1550030, 2015.

[4] Carlier M.F., and Pantaloni D. Control of actin dynamics in cell motility, J. Mol. Biol., 269(4):459-467, 1997.

[5] Egelman E.H. The structure of F-actin, J. Muscle Res. Cell. Motil., 6(2):129-151, 1985.

[6] Etienne-Manneville S. Actin and microtubules in cell motility: which one is in control? Traffic, $5(7): 470-477,2004$

[7] Hill T.L. Microfilament or microtubule assembly or disassembly against a force, Proc. Natl. Acad. Sci. U.S.A., 78(9):5613-5617, 1981.

[8] Kornai A. Euclidean Automata. In: M. Waser (ed.), Implementing Selves with Safe Motivational Systems and Self-Improvement. Proc. AAAI Spring Symposium, AAAI Press, 2014, 25-30.

[9] Le Clainche C., and Carlier M. Regulation of actin assembly associated with protrusion and adhesion in cell migration, Physiol. Rev., 88(2):489-513, 2008.
[10] Maly I.V., and Borisy G.G. Self-organization of a propulsive actin network as an evolutionary process, Proc. Natl. Acad. Sci. U.S.A., 98(20):11324-11329, 2001.

[11] Mayne R., Adamatzky A., and Jones J. On the role of the plasmodial cytoskeleton in facilitating intelligent behavior in slime mold Physarum polycephalum, Communicative $\&$ integrative biology, 8(4): e1059007, 2015.

[12] McGough A. F-actin-binding proteins, Curr. Opin. Struct. Biol., 8(2):166-176, 1998.

[13] Mogilner A., and Oster G. Cell motility driven by actin polymerization, Biophys. J., 71(6):3030-3045, 1996.

[14] Moore P.B., Huxley H.E., and DeRosier D.J. Three-dimensional reconstruction of F-actin, thin filaments and decorated thin filaments, J. Mol. Bioli., 50(2):279-295, 1970.

[15] Mooseker M.S., and Tilney L.G. Organization of an actin filament-membrane complex. Filament polarity and membrane attachment in the microvilli of intestinal epithelial cells, J. Cell Biol., 67(3):725-743, 1975.

[16] Pollard T.D., and Cooper J.A. Actin, a central player in cell shape and movement, Science, 326(5957):1208-1212, 2009.

[17] Schumann A., Woleński J. Two Squares of Oppositions and Their Applications in Pairwise Comparisons Analysis, Fundamenta Informaticae,144(3-4):241-254, 2016.

[18] Schumann A. Conventional and unconventional reversible logic gates on Physarum polycephalum, International Journal of Parallel, Emergent and Distributed Systems, 2015; doi: 10.1080/17445760.2015.1068775

[19] Schumann A. p-Adic valued logical calculi in simulations of the slime mould behaviour, Journal of Applied Non-Classical Logics, 2015, doi: 10.1080/11663081.2015.1049099

[20] Schumann A. Towards context-based concurrent formal theories, Parallel Processing Letters, 25(1):1540008, 2015.

[21] Shirakawa T., Sato H., Ishiguro S. Constrcution of living cellular automata using the Physarum plasmodium, Int. J. Gen. Syst. 44:292-304, 2015.

[22] Shirakawa T., Yokoyama K., Yamachiyo M., Gunji Y.-P., Miyake Y. Multi-scaled adaptability in motility and pattern formation of the Physarum plasmodium, Int. J. of Bio-Inspired Computation, 4:131-138, 2012.

[23] Siccardi S., Tuszynski J.A., and Adamatzky A. Boolean gates on actin filaments, Physics Letters A, 380(1):88-97, 2016.

[24] Siccardi S., and Adamatzky A. Logical Gates Implemented by Solitons at the Junctions Between One-Dimensional Lattices, International Journal of Bifurcation and Chaos, 26(6):1650107, 2016.

[25] Siccardi S., and Adamatzky A. Actin quantum automata: Communication and computation in molecular networks, Nano Communication Networks, 6(1):15-27, 2015. 